\title{
Pregnancy in Systemic Sclerosis: Results of a Systematic Review and Metaanalysis
}

\author{
Jelena Blagojevic, Khitam Abdullah AlOdhaibi, Aly M. Aly, Silvia Bellando-Randone, \\ Gemma Lepri, Cosimo Bruni, Alberto Moggi-Pignone, Serena Guiducci, Federico Mecacci, \\ Marco Matucci-Cerinic, and Daniel E. Furst
}

\begin{abstract}
Objective. Through a systematic literature search (SLR) and metaanalysis, to determine maternal and fetal outcomes in pregnancies involving systemic sclerosis (SSc), to analyze the effect of pregnancy on disease activity, and to examine predictors of fetal and maternal outcomes.

Methods. An SLR was performed for articles on SSc and pregnancy published between 1950 and February 1,2018. Reviewers double-extracted articles to obtain agreement on $>95 \%$ of predefined critical outcomes.

Results. Out of 461 publications identified, 16 were included in the metaanalysis. The metaanalysis showed that pregnancies involving SSc were at higher risk of miscarriage (OR 1.6, 95\% CI 1.22-2.22), fetuses with intrauterine growth retardation (IUGR; OR 3.2, 95\% CI 2.21-4.53), preterm births (OR 2.4, 95\% CI 1.14-4.86), and newborns with low birth weight (OR 3.8, 95\% CI 2.16-6.56). Patients with SSc had a 2.8 times higher chance of developing gestational hypertension (HTN; OR 2.8, 95\% CI 2.28-3.39) and a 2.3 times higher chance of cesarean delivery compared to controls (OR 2.3, 95\% CI 1.37-3.8). The definitions of disease worsening/new visceral organ involvement were too inexact to have any confidence in the results, although worsening or new disease manifestations during pregnancy in $44 / 307$ cases $(14.3 \%)$ and 6 months postpartum in 32/306 cases $(10.5 \%)$ were reported. The data did not permit definition of predictors of disease progression and of maternal and fetal outcomes.

Conclusion. Pregnancies involving SSc have increased frequency of miscarriages, IUGR, preterm deliveries, and newborns with low birth weight compared to healthy controls. Women with SSc were more prone to develop gestational HTN and to undergo cesarean delivery. Disease manifestations seem to remain stable or improve in most patients. (First Release February 15 2020; J Rheumatol 2020;47:881-7; doi:10.3899/jrheum.181460)
\end{abstract}

Key Indexing Terms: PREGNANCY

From the Department of Experimental and Clinical Medicine, University of Florence, and the Department of Geriatric Medicine, Division of Rheumatology and Scleroderma Unit, Azienda Ospedaliero Universitaria Careggi (AOUC), Florence, Italy; Department of Family Medicine, King Faisal Specialist Hospital and Research Centre, Riyadh, Saudi Arabia; Faculty of Medicine, Alexandria University, Alexandria, Egypt; Department of Emergency Medicine, Division of Medicine IV AOUC; Department of Maternal-Neonatal Caref, Careggi University Hospital, Florence, Italy; University of California at Los Angeles, Los Angeles, California; University of Washington, Seattle, Washington, USA;

University of Florence, Florence, Italy.

J. Blagojevic, MD, PhD, Department of Experimental and Clinical Medicine, University of Florence, and Department of Geriatric Medicine, Division of Rheumatology and Scleroderma Unit, AOUC; K.A. AlOdhaibi, $M D$, Department of Family Medicine, King Faisal Specialist Hospital and Research Centre; A.M. Aly, MBBch, Faculty of Medicine, Alexandria University; S. Bellando-Randone, $M D, P h D$, Department of Experimental and Clinical Medicine, University of Florence, and Department of Geriatric Medicine, Division of Rheumatology and Scleroderma Unit, AOUC; G. Lepri, MD, Department of Experimental and Clinical Medicine, University of Florence, and Department of Geriatric Medicine, Division of Rheumatology and Scleroderma Unit, AOUC; C. Bruni, MD, Department of Experimental and Clinical Medicine, University of Florence, and Department of Geriatric Medicine, Division of Rheumatology and Scleroderma Unit, AOUC; A. Moggi-Pignone, MD, $P h D$, Department of Experimental and Clinical Medicine, University of Florence, and Department of Emergency Medicine, Division of Medicine

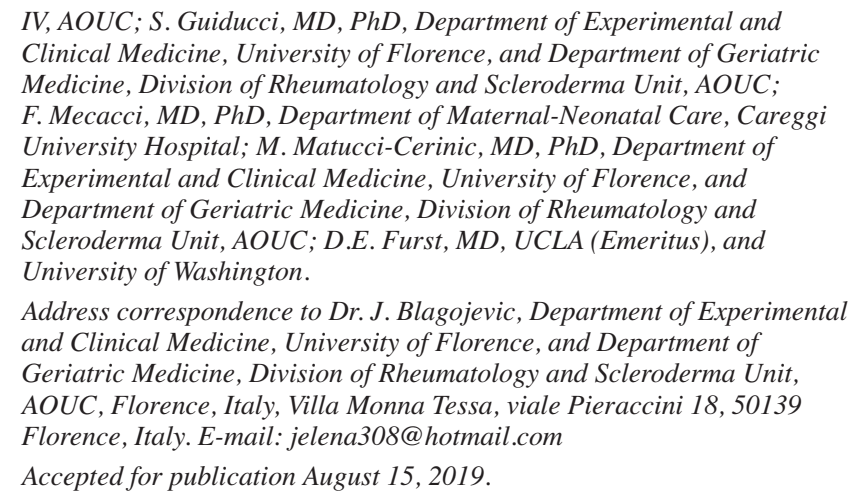
Clinical Medicine, University of Florence, and Department of Geriatric Medicine, Division of Rheumatology and Scleroderma Unit, AOUC; F. Mecacci, MD, PhD, Department of Maternal-Neonatal Care, Careggi University Hospital; M. Matucci-Cerinic, MD, PhD, Department of Experimental and Clinical Medicine, University of Florence, and Department of Geriatric Medicine, Division of Rheumatology and Scleroderma Unit, AOUC; D.E. Furst, MD, UCLA (Emeritus), and University of Washington.

Address correspondence to Dr. J. Blagojevic, Department of Experimental and Clinical Medicine, University of Florence, and Department of Geriatric Medicine, Division of Rheumatology and Scleroderma Unit, AOUC, Florence, Italy, Villa Monna Tessa, viale Pieraccini 18, 50139 Florence, Italy.E-mail: jelena308@hotmail.com

Accepted for publication August 15, 2019.

Systemic sclerosis ( $\mathrm{SSc}$ ) is a complex autoimmune disease characterized by small-vessel vasculopathy, immune system activation, and fibrosis ${ }^{1}$, leading to skin and internal organ involvement. SSc has a strong female predominance ${ }^{2}$ and women can be affected in their childbearing age, potentially leading to adverse pregnancy outcomes. Therefore, the ability to carry a pregnancy to a safe conclusion is important to the quality of life and well-being of patients with $\mathrm{SSc}^{3}$. 
Several studies have been reported of fetal and maternal outcomes in patients with SSc, mainly retrospective and/or questionnaire-based. Earlier studies, published before 1990, showed high rates of pregnancy-related maternal mortality and morbidity and an increased frequency of miscarriages and fetal loss ${ }^{4,5,6}$. More recently, a better outcome has been described with mostly successful pregnancies, but there have also been reports of an increased risk of premature births $7,8,9,10,11$, intrauterine growth restriction (IUGR) $)^{10,12}$, and low birth weight ${ }^{7,8,10}$.

Data on maternal adverse outcomes are limited. Ismail, et al reported an increased prevalence of preeclampsia in a small observational study of 20 pregnant patients with SSc and 20 age-matched healthy controls ${ }^{13}$. This finding was not confirmed in an Italian multicenter study including 109 pregnancies involving $\mathrm{SSc}^{10}$.

No significant changes in maternal SSc disease activity have been described during pregnancy $7,14,15$. Still, SSc is considered a condition causing increased risk of pregnancy complications and maternal and fetal morbidity. In particular, higher risk for obstetric complications has been seen in patients with early diffuse cutaneous SSc $(<4$ yrs of disease duration $)^{7}$, those who are antitopoisomerase I or anti-RNA polymerase III-positive ${ }^{16,17}$, and those using corticosteroids ${ }^{10}$. To date, no clear predictors of adverse fetal and maternal outcome have been identified.

The aim of our study was to assess maternal and fetal outcomes in pregnancies with SSc, to analyze the effect of pregnancy on SSc disease activity, and to determine whether there are predictors of fetal and maternal outcomes. The study was conducted with a Preferred Reporting Items for Systematic reviews and Meta-Analyses (PRISMA)-driven systematic review and a metaanalysis of the published literature.

\section{MATERIALS AND METHODS}

A systematic literature search (SLR), according to the PRISMA recommendations ${ }^{18}$, was performed in 4 different databases: PubMed, Cochrane, EMBASE, and Web of Science, for articles published in peer-reviewed journals between 1950 and February 1, 2018, in English and Italian. All articles reporting on SSc and pregnancy were examined. The keyword search terms included "systemic sclerosis OR scleroderma, AND pregnancy".

A written protocol for data search and extraction was prepared (Supplementary Data File 1, available from the authors on request). Studies were eligible if they met the following inclusion criteria: pregnancies occurred after disease onset, the article included solely patients with SSc or the patients with SSc and their outcomes could be separated from patients with other conditions, and there was at least 1 clinical outcome of interest. Studies could be randomized clinical trials, retrospective or observational studies, registries, case series with and without controls, and case reports with $\geq 10$ pregnancies. The exclusion criteria were non-human studies, articles including only patients with overlap syndrome by American College of Rheumatology (ACR)/European League Against Rheumatism classification criteria, and case reports including $<10$ pregnancies.

Full-text articles were included, as were abstracts if they contained enough of the clinical data required for the SLR. The bibliographies of the articles were also evaluated for additional references. Reviews were listed and examined for articles in the bibliography that were not surfaced during the SLR; the reviews per se were not extracted.

Ethics approval was not required because the study did not include human and/or animal subjects.

A dedicated research librarian performed the systematic literature search. Two reviewers (KAA and JB) screened all articles for inclusion/exclusion criteria and agreement was obtained on all of them. The same 2 reviewers double-extracted articles, with a third reviewer (DF) resolving differences. The reviewers' purpose was to (1) obtain agreement on $>95 \%$ of predefined critical outcomes and $>90 \%$ of the other variables; (2) maintain quality assurance; and (3) screen and extract data.

The studies that met inclusion criteria and were not excluded were extracted in an electronic database for descriptive variables, SSc disease activity, relevant clinical outcomes, and for effect of pregnancy on maternal and fetal outcomes. Data on pregnancies in controls were also extracted, where available.

Fetal outcomes. Fetal outcomes were miscarriage (generally fetal loss $<20$ weeks), stillbirth/perinatal death (generally fetal loss $\geq 20$ weeks or $<4$ weeks of life), IUGR (estimated fetal weight $<10$ th percentile), premature delivery (birth occurring before $<37$ th week of pregnancy), low birth weight $(<2500 \mathrm{~g}$ at birth), small for gestational age (weight $<10$ th percentile for age), and congenital malformations. We included only spontaneous abortions among miscarriages, where this information was available.

Maternal outcomes. Maternal outcomes were maternal death, eclampsia or preeclampsia, and their components. The rate of cesarean deliveries was also assessed. In addition, rates of gestational hypertension (HTN) and diabetes were recorded (details in Supplementary Table 1, available from the authors on request).

Because none of the selected articles specifically reported SSc activity indices, disease activity was determined by assessing the proportion of pregnancies in which disease/single organ manifestations worsened/ improved/remained stable during pregnancy and within 6 months postpartum, as reported in the included studies. The occurrence of new organ manifestations was considered as worsening. The number of studies in which the disease/organ manifestations worsened/improved/remained stable during and after pregnancy was also listed, to allow understanding of the strength of the data analyzed. Definitions of disease activity and organ involvement, where available, are reported in Supplementary Data File 2 (available from the authors on request).

Analysis. For the continuous variables, descriptive analysis included means and SD; for the categorical variables, proportions and percentages have been reported. Quality and risk of bias assessment was performed by JB using the Newcastle-Ottawa Scale (NOS) ${ }^{19}$.

Because not all the studies reported all the maternal and fetal outcomes, separate metaanalyses were performed for each outcome. Publication bias was assessed by visual inspection of funnel plots.

In addition, other potential sources of bias (recall bias for studies based on questionnaire, lack of a uniform classification of SSc, and definition of maternal and fetal outcomes across the studies) were considered (Supplementary Data File 2, available from the authors on request) and causes of publication bias were investigated.

The heterogeneity across the studies was assessed by Woolf's test (there was significant heterogeneity for the $\mathrm{p}$ values $<0.005$ ). The random effects (DerSimonian-Laird) metaanalysis model was used to estimate the pooled effect for results with significant heterogeneity. The fixed effects (Mantel-Haenszel) metaanalysis model was applied if there was no heterogeneity across the studies analyzed. Each metaanalysis provides a pooled OR and its 95\% CI. Data were analyzed by R Studio version 1.2.1.

\section{RESULTS}

The search identified 461 publications. Sixteen studies meeting the inclusion criteria were included in the data SLR and metaanalysis (Supplementary Figure 1, available from the authors on request). 
The summary of the characteristics of 16 included studies is shown in Supplementary Table 2 (available from the authors on request).

Seven articles had no controls and 1 article was only in summary form, so these articles were not quantifiable by the NOS criteria. In the other 8 , one was of high quality, 4 were of medium quality, and 3 were of low quality (Supplementary Table 3, available from the authors on request).

Funnel plots describing publication bias are shown in Supplementary Figures 2-10 (available from the authors on request). It was not possible to assess the publication bias for articles reporting maternal death and eclampsia because so few studies were available. The year of publication did not affect the publication bias. A total of 1403 pregnancies in the SSc group and 12.2 million pregnancies in the healthy controls group were analyzed. Clinical characteristics of pregnant patients with SSc are shown in Supplementary Table 4. Disease duration was just over 7 years. All of them had skin involvement before pregnancy (where reported) and $47.5 \%$ had diffuse cutaneous disease. Reports of visceral involvement were uncommon.

Disease treatment during pregnancy could not be reliably ascertained because there were so few patients in whom it was described (30/461 patients), and even there the definitions were variable and inexact.

Description of maternal and fetal outcomes in patients with SSc across single studies is shown in Supplementary Table 5 (available from the authors on request).

The results of the metaanalyses of the studies reporting different fetal outcomes are shown in Figure 1, Figure 2, Figure 3, and Figure 4, and Supplementary Figures 11, 12, and 13 (available from the authors on request). There was no significant heterogeneity across the studies, except among the studies on premature deliveries.

SSc pregnancies ended in miscarriages 1.6 times more often than control pregnancies (OR 1.6, 95\% CI 1.22-2.22; Figure 1). Fetuses of women with $\mathrm{SSc}$ had a 3.2 times higher chance of having IUGR (OR 3.2, 95\% CI 2.21-4.53) than did controls
(Figure 2). Newborns of mothers with SSc had a 2.4 times higher risk of being premature deliveries (OR 2.4, 95\% CI 1.14-4.86; Figure 3) and a 3.8 times higher chance of having low birth weight (OR 3.8, 95\% CI 2.16-6.56; Figure 4).

The results of the metaanalyses of the studies reporting different maternal outcomes are shown in Supplementary Figures 14, 15, and 16 (available from the authors on request). There was no increased risk of preeclampsia in pregnant women with SSc compared to controls (Figure 5). Patients with SSc had a 2.8 times higher chance of developing gestational HTN (OR 2.8, 95\% CI 2.28-3.39; Supplementary Figure 14) but not diabetes (Supplementary Figure 15), and a 2.3 times higher chance of having cesarean delivery compared to controls (OR 2.3, 95\% CI 1.37-3.8; Supplementary Figure 16).

There were too few articles reporting on maternal death and eclampsia in patients with SSc to perform metaanalyses on those outcomes.

Disease activity remained stable or improved in most patients with SSc. One or more disease manifestations worsened or appeared during pregnancy in 44/307 (14.3\%) cases. In 32/306 (10.5\%) pregnancies, SSc manifestations worsened or appeared during the 6-month postpartum period. In 2 reported cases, this worsening led to maternal death from scleroderma renal crisis ${ }^{1}$ and aspiration pneumonia complicated by respiratory and multiorgan failure ${ }^{1}$.

Data on worsening and improvement of SSc and its single-organ manifestations, extrapolated from the studies included in the metaanalysis, are summarized in Table 1 (more details in Supplementary Tables 6 and 7, available from the authors on request).

It is important to note that, except for overall disease activity, data on visceral involvement during and after pregnancy were documented in $<50 \%$ of the studies (Supplementary Tables 6 and 7, available from the authors on request) and the definitions were variable and inexact. Therefore, it is not possible to confidently ascribe specific visceral changes that might have occurred.

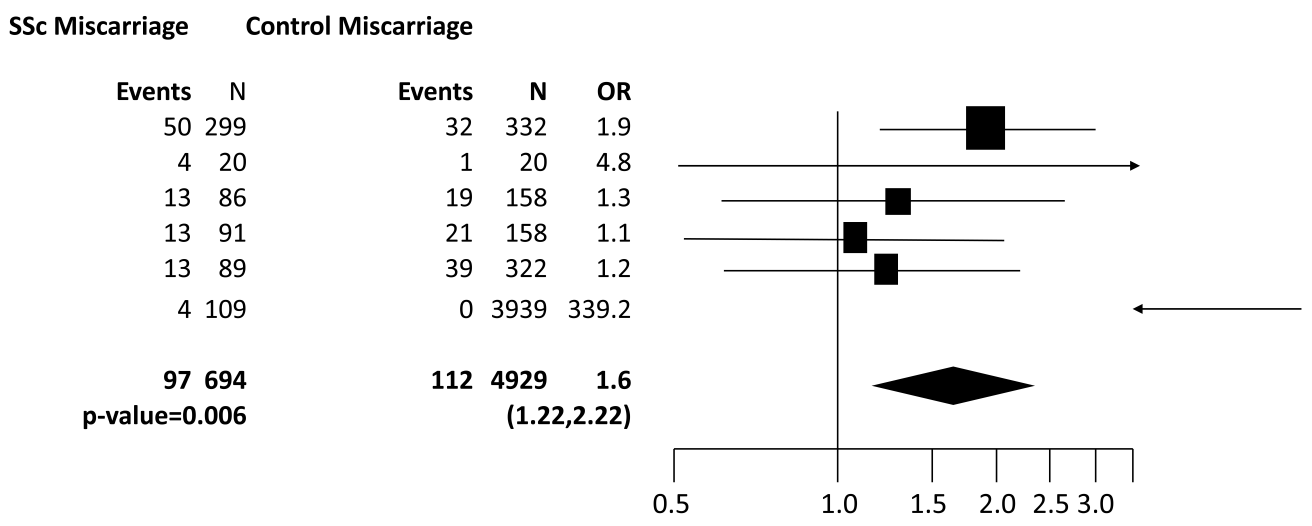

Figure 1. Comparison of miscarriages in patients with SSc and control subjects. SSc: systemic sclerosis. Personal non-commercial use only. The Journal of Rheumatology Copyright (C) 2020. All rights reserved. 
SSc IUGR

Study

Chakravarty EF (ref 12)

Ismail $F$ (ref 13)

Taraborelli M (ref 10)

Summary

Test for heterogeneity

\section{Control IUGR}

Events N Events

$27 \quad 504$

220

6109

35633

p-value $=0.48$
190400

\begin{tabular}{rr}
$\mathbf{N}$ & OR \\
11200000 & 3.3 \\
20 & 1.0 \\
3939 & 3.9 \\
& \\
11203959 & $\mathbf{3 . 2}$ \\
\hline
\end{tabular}

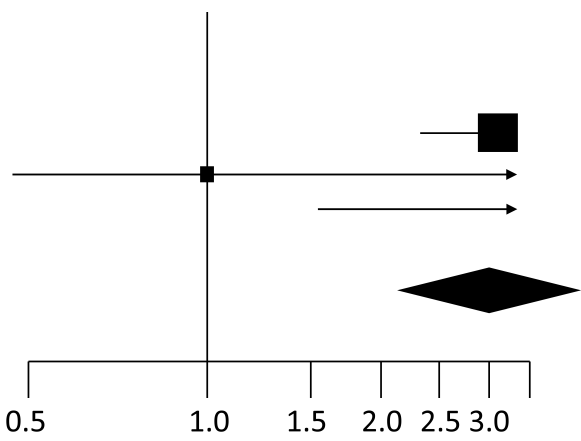

Figure 2. Comparison of intrauterine growth retardation in SSc patients and control subjects. SSc: systemic sclerosis; IUGR: intrauterine growth retardation.

\begin{tabular}{|c|c|c|c|c|}
\hline \multicolumn{2}{|c|}{ SSc premature delivery } & Control premature delivery & \multirow[b]{2}{*}{$\mathbf{N}$} & \multirow[b]{2}{*}{ OR } \\
\hline Study & Events $\mathrm{N}$ & $\begin{array}{l}\text { Events } \\
\text { Even }\end{array}$ & & \\
\hline Chakravarty EF (ref 12) & 6504 & 414400 & 11200000 & 0.31 \\
\hline Chen JS (ref 11) & 1653 & 65268 & 991292 & 6.14 \\
\hline Ismail F (ref 13) & 20 & 2 & 20 & 3.86 \\
\hline Steen VD 1989 (ref 29) & 86 & 8 & 158 & 1.66 \\
\hline Steen VD, 1999 (ref 7) & 91 & 8 & 158 & 6.34 \\
\hline Steen VD, Medsger TA 1999 (ref 8) & 13 & 25 & 322 & 2.03 \\
\hline Taraborelli M (ref 10) & 50109 & 940 & 3939 & 2.70 \\
\hline Summary & 121952 & 480651 & 12195889 & 2.4 \\
\hline Test for heterogeneity & $p$-value $=0$ & & $(1.14$ & 4.86) \\
\hline
\end{tabular}

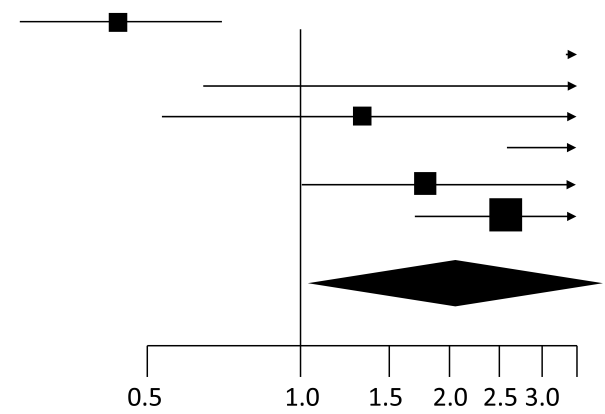

Figure 3. Comparison of premature deliveries in patients with SSc and control subjects. SSc: systemic sclerosis.

\section{SSc low birth weights Control low birth weights}

\section{Study}

Steen VD, 1989 (ref 29)

Steen VD, 1999 (ref 7)

Steen VD, Medsger TA 1999 (ref 8)

Taraborelli M (ref 10)

\section{Summary}

Test for heterogeneity

$\begin{array}{rr}\text { Events } & N \\ 9 & 86 \\ 3 & 91 \\ 10 & 89 \\ 5 & 109\end{array}$

p-value $=0.80$

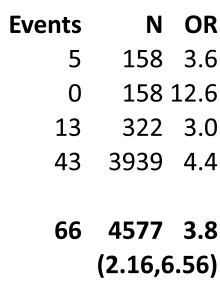

$(2.16,6.56)$

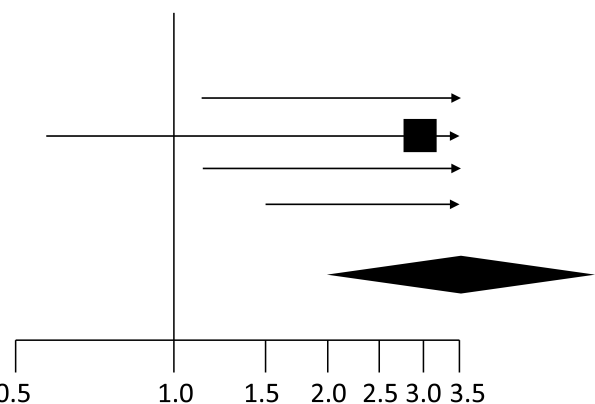

Figure 4. Comparison of newborns with low birth weight in patients with SSc and control subjects. SSc: systemic sclerosis.

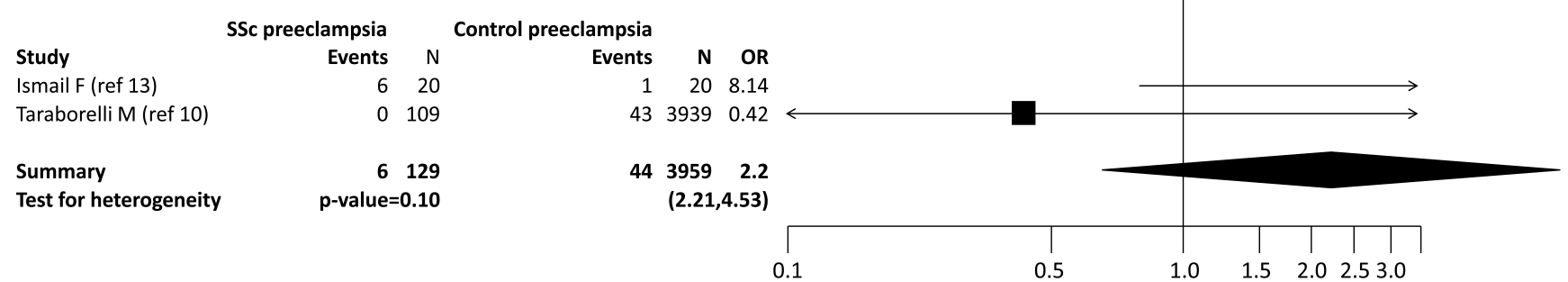

Figure 5. Comparison of preeclampsia in patients with SSc and control subjects. SSc: systemic sclerosis. 
Table 1. Pregnancy and SSc activity: worsening and improvement.

\begin{tabular}{|c|c|c|c|c|}
\hline \multirow[t]{2}{*}{ Variables } & \multicolumn{2}{|c|}{ SSc Worsening } & \multicolumn{2}{|c|}{ SSc Improvement } \\
\hline & Pregnancy & $\begin{array}{l}\text { Postpartum (within } 6 \text { mos } \\
\text { after delivery) }\end{array}$ & Pregnancy & $\begin{array}{c}\text { Postpartum (within } 6 \text { mos } \\
\text { after delivery) }\end{array}$ \\
\hline $\begin{array}{l}\text { Peripheral vascular (RP and/or } \\
\text { digital ulcers) }\end{array}$ & $2 / 12(16.6)$ & 18/111 (16.2) & $77 / 220(35)$ & $22 / 109(20.2)$ \\
\hline Skin & $3 / 103(2.9)$ & $30 / 220(13.6)$ & $4 / 20(20)$ & NA \\
\hline ILD & $2 / 118(1.7)$ & NA & $0 / 86(0)$ & NA \\
\hline PAH & $1 / 20(5)$ & NA & NA & NA \\
\hline $\mathrm{SRC}$ & 6/197 (3) & $3 / 286(1)$ & NA & $0 / 86(0)$ \\
\hline Heart condition & 3/177 (1.7) & $2 / 109(1.8)$ & $0 / 86(0)$ & NA \\
\hline
\end{tabular}

Values are $\mathrm{n} / \mathrm{N}(\%)$. Percentages are relative to the number of pregnancies for which data are available in single studies. SSc: systemic sclerosis; RP: Raynaud phenomenon; ILD: interstitial lung disease; PAH: pulmonary arterial hypertension; SRC: scleroderma renal crisis; NA: not available.

\section{DISCUSSION}

To our knowledge, this is the first systematic PRISMA-based review and metaanalysis that specifically addresses maternal and fetal outcomes and the effect of pregnancy on disease activity in pregnancies that occurred after the onset of SSc.

There are a number of review articles on pregnancies in autoimmune connective tissue diseases, but few of them specifically addressed pregnancies involving SSc 3 ,12,14,15,17,20-26. None were systematic literature reviews, and several were done more than 20 years ago when diagnostic criteria were not as uniform ${ }^{14,20,21,22,23}$ and the number of studies available for review were even fewer than today.

For context, we compared maternal and fetal outcomes in pregnancies involving SSc versus healthy women/general obstetric populations by quantitative analysis of published data. We also examined disease activity in the 6-month postpartum period for the first time, to our knowledge.

Our systematic literature review and metaanalysis indicate that pregnancies involving SSc have an increased prevalence of miscarriages, IUGR, preterm births, and newborns with low birth weight, but not small for gestational age, compared to pregnancies in healthy controls.

The review by Sobanski, et al reported that the rates of miscarriages were not increased in pregnancies of patients with $\mathrm{SSc}^{17}$. However, they included studies of pregnancies that occurred both before and after disease onset, and no metaanalysis was performed. Moreover, other maternal and fetal outcomes were not analyzed in detail.

Betelli, et al recently published a review focused on maternal and fetal outcomes in pregnancies occurring after SSc onset ${ }^{3}$. They concluded that the risk of miscarriage in women who were followed as high-risk pregnancies was comparable to that of the healthy population ${ }^{3}$. However, this review was not based on a systematic literature research, no metaanalysis was performed, and no conclusion was made for women with SSc who were not followed as high-risk pregnancies.
On the other hand, an older review by Scarpinato and Mackenzie, which included only case reports and 1 case series published from 1932 to 1984 , reported a near-term fetal death rate of $23 \%$ in SSc pregnancies ${ }^{22}$. Their data are probably skewed by the character of the data (case reports and series). The rate of late fetal loss was low in our metaanalysis $(4.4 \%)$.

Regarding other fetal outcomes, premature births and IUGR were also more frequent in pregnancies involving SSc according to the Betelli, et al review ${ }^{3}$. A reason for these adverse fetal outcomes could be the impairment of placental vascularization as part of SSc-related vasculopathy ${ }^{27}$.

Scarpinato and Mackenzie reported high maternal mortality $(15 \%$ of 82 women with SSc included in their review $)^{22}$. The main cause of maternal death was accelerated HTN (most likely scleroderma renal crisis, although not specified). However, the author noted that case reports were likely biased toward more severe outcomes because pregnancies with normal outcomes were probably reported less frequently compared to those leading to maternal or fetal loss. In our literature review, scleroderma renal crisis was often not mentioned, but when mentioned was documented in only $1-3 \%$ of pregnancies. This is not greatly different from the literature in a metaanalysis of the non-pregnant SSc population $(4 \%)^{28}$. This comparison must be viewed in the context of the lack of mention of scleroderma renal crisis in our literature review, thus making it possible that the 1-3\% represents underreporting.

The data regarding maternal mortality were too scarce to perform a metaanalysis. There were 3 studies (all published before 2000) that reported maternal death in 4 patients $(1.3 \%)^{4,7,29}$. The causes of the deaths were scleroderma renal crisis (1), aspiration pneumonia complicated by respiratory and multiorgan failure (1), viral pneumonia (1), and bronchopneumonia complicated by congestive heart failure (1).

Our metaanalysis indicates that women with SSc had a greater chance of developing gestational HTN. However,

Personal non-commercial use only. The Journal of Rheumatology Copyright (c) 2020. All rights reserved. 
unexpectedly, they were not at increased risk of preeclampsia. This was not expected because pregnancy-induced HTN, preeclampsia, and SSc are all related through endothelial dysfunction, raising the expectation that HTN and preeclampsia would be correlated ${ }^{1,30}$. The reasons that pregnancies involving SSc seem to be at higher risk of gestational HTN but not of preeclampsia have to be investigated.

Our results show that cesarean deliveries were more frequent in pregnancies with SSc compared to controls. It is possible that patients with SSc are considered at risk of disease-related musculoskeletal alterations of the pelvic region and thus were referred for cesarean deliveries more frequently.

We attempted to evaluate the effect of pregnancy on SSc disease activity, based mainly on the descriptions available in single articles and reported as patients' or authors' judgment of disease activity. None of the studies used standardized disease activity indices and the descriptions were variable and often very inexact. The analysis of available data indicates that overall disease activity, however defined, appeared to remain stable or improved during pregnancy in most cases. Peripheral vascular manifestations such as Raynaud phenomenon, digital ulcers, and skin and articular involvement appeared to remain stable or improve during pregnancy, as already suggested by previous reviews on this topic ${ }^{17,25}$.

The same limitations applicable regarding disease activity during pregnancy also applied to the 6-month postpartum period. Within those limitations, inherent in the data, overall disease activity and peripheral vascular and skin manifestations appeared to remain stable or improved in the majority of the cases.

Unfortunately, we could not perform an analysis of the effect of pregnancy on other SSc manifestations, such as interstitial lung disease (ILD), scleroderma renal crisis, heart involvement, or pulmonary arterial HTN (PAH), because of the small number of cases described in the articles.

Betelli, et al reported in their recent review that overall disease activity was generally stable during pregnancy ${ }^{3}$. According to these authors, cardiopulmonary involvement, including $\mathrm{PAH}$, also remained stable, but this statement was based only on data of the IMPRESS study (an Italian multicenter retrospective study of 109 pregnancies involving SSc followed from 2000 to 2011$)^{3}$.

It has been reported that patients with early diffuse SSc and severe organ involvement are at higher risk of developing adverse maternal and fetal outcomes $8,16,17$. In our pooled analysis of the literature, half of the patients had the diffuse cutaneous subset, the mean disease duration was 7 years, and ILD was present in $17 \%$ patients before pregnancy. Only $0.8 \%$ of patients had PAH and $1.3 \%$ had a history of scleroderma renal crisis before becoming pregnant, thus making it likely that these data represent underreporting.

One of the objectives of our study was to investigate predictors of maternal and fetal outcomes. To make predictions there needed to be full descriptions of disease manifestations during pregnancy as well as complete descriptions of outcomes postpartum. In addition, the medications used during and after pregnancy needed to be complete. Unfortunately, none or very few of these data were available in the literature. The data of the IMPRESS study showed that corticosteroid use was associated with preterm deliveries, whereas folic acid supplementation and anti-Scl-70 antibodies were protective ${ }^{10}$, but we could not examine these factors in the literature because they were rarely reported. Therefore, we were not able to construct a predictive model

Our study has significant strengths. It was a thorough systematic literature review and metaanalysis, the first for pregnancies during SSc to our knowledge, and was documented using PRISMA principles (Supplementary Data File 3, available from the authors on request). Data were double-extracted throughout, minimizing errors. Methods were transparent and our interpretations were conservative, allowing the reader clarity and (it is hoped) making our conclusions credible.

Our study has some limitations. All the articles were retrospective and most included a very small number of subjects, although this shortcoming will be difficult to overcome because pregnancies appear to be infrequent in women with SSc (personal observation). The metaanalyses were very limited and in some cases were based only on few studies.

The studies were carried out over nearly 50 years and the definition of SSc changed, so it was not clear that the patient populations were comparable. However, the 1980 and 2013 SSc definitions are sufficiently similar ${ }^{31,32}$. Also, there was no uniform definition of maternal and fetal outcomes, although there seemed to be general agreement (e.g., miscarriage defined as fetal loss before 20 weeks). There was probably a tendency to report pregnancies with worse outcomes, especially in the older case series; therefore, more contemporary studies are needed. We compared pregnancies involving SSc to normal controls; it would be instructive to compare SSc pregnancies to those occurring during other connective tissue diseases. Unfortunately, these data were simply not available within the extracted SSc studies. Finally, there were incomplete data available on patient characteristics and treatments as well as measures of disease activity, so we could not credibly define the prevalence of worsening or internal organ involvement, or construct a predictive model.

Pregnancy seems to be safe for the mother who has SSc, although there seems to be an increased risk of miscarriage, IUGR, premature delivery, newborns with low birth weight, and gestational HTN.

As far as could be determined with incomplete and variable data, SSc appeared to remain stable or improved in the majority of patients. About $10-15 \%$ of the patients had worsening or new disease manifestations during and within

Personal non-commercial use only. The Journal of Rheumatology Copyright $\subset$ 2020. All rights reserved. 
6 months of completing pregnancy. We could not assess the effect of SSc on major organ involvement, and predictors of negative maternal and fetal outcomes could not be assessed because data were lacking.

\section{REFERENCES}

1. Varga J, Trojanowska M, Kuwana M. Pathogenesis of systemic sclerosis: recent insights of molecular and cellular mechanisms and therapeutic opportunities. J Scleroderma Relat Disord 2017; 2:137-52.

2. Mayes MD, Lacey JV Jr, Beebe-Dimmer J, Gillespie BW, Cooper B, Laing TJ, et al. Prevalence, incidence, survival, and disease characteristics of systemic sclerosis in a large US population. Arthritis Rheum 2003;48:2246-55.

3. Betelli M, Breda S, Ramoni V, Parisi F, Rampello S, Limonta M, et al. Pregnancy in systemic sclerosis. J Scleroderma Relat Disord 2018;3:21-9.

4. Slate WG, Graham AR. Scleroderma and pregnancy. Am J Obstet Gynecol 1968;101:335-41.

5. Giordano M, Valentini G, Lupoli S, Giordano A. Pregnancy and systemic sclerosis. Arthritis Rheum 1985;28:237-8.

6. Black CM. Systemic sclerosis and pregnancy. Baillieres Clin Rheumatol 1990;4:105-24.

7. Steen VD. Pregnancy in women with systemic sclerosis. Obstet Gynecol 1999;94:15-20.

8. Steen VD, Medsger TA Jr. Fertility and pregnancy outcome in women with systemic sclerosis. Arthritis Rheum 1999;42:763-8.

9. Chung L, Flyckt RL, Colon I, Shah AA, Druzin M, Chakravarty EF. Outcome of pregnancies complicated by systemic sclerosis and mixed connective tissue disease. Lupus 2006;15:595-9.

10. Taraborelli M, Ramoni V, Brucato A, Airò P, Bajocchi G, Bellisai F, et al; IMPRESS Investigators. Brief report: successful pregnancies but a higher risk of preterm births in patients with systemic sclerosis: an Italian multicenter study. Arthritis Rheum 2012;64:1970-7.

11. Chen JS, Roberts CL, Simpson JM, March LM. Pregnancy outcomes in women with rare autoimmune diseases. Arthritis Rheumatol 2015;67:3314-23.

12. Chakravarty EF, Khanna D, Chung L. Pregnancy outcomes in systemic sclerosis, primary pulmonary hypertension, and sickle cell disease. Obstet Gynecol 2008;111:927-34.

13. Ismail F, Abdel-Azeem MI, Abd El-Haleem H, Mohammed AB, Katta MA, Mahmoud A, et al. Feto-maternal outcome in patients with systemic sclerosis. Egypt Rheumatologist 2013;35:101-6.

14. Black CM, Stevens WM. Scleroderma. Rheum Dis Clin North Am 1989;15:193-212.

15. Rueda de León Aguirre A, Ramírez Calvo JA, Rodríguez Reyna TS. Comprehensive approach to systemic sclerosis patients during pregnancy. Reumatol Clin 2015;11:99-107.
16. Tincani A, Dall'Ara F, Lazzaroni MG, Reggia R, Andreoli L. Pregnancy in patients with autoimmune disease: a reality in 2016. Autoimmun Rev 2016;15:975-7.

17. Sobanski V, Launay D, Depret S, Ducloy-Bouthors AS, Hachulla E. Special considerations in pregnant systemic sclerosis patients. Expert Rev Clin Immunol 2016;12:1161-73.

18. Moher D, Liberati A, Tetzlaff J, Altman DG; PRISMA Group. Preferred reporting items for systematic reviews and meta-analyses: the PRISMA statement. Int J Surg 2010;8:336-41.

19. Wells GA, Shea B, O'Connell D, Peterson J, Welch V, Losos M, et al. The Newcastle-Ottawa Scale (NOS) for assessing the quality of nonrandomised studies in meta-analyses [Internet. Accessed January 9, 2020.] Available from: www.ohri.ca/programs/clinical_ epidemiology/oxford.asp

20. Goplerud CP. Scleroderma. Clin Obstet Gynecol 1983;26:587-91.

21. Ballou SP, Morley JJ, Kushner I. Pregnancy and systemic sclerosis. Arthritis Rheum 1984;27:295-8.

22. Scarpinato L, Mackenzie AH. Pregnancy and progressive systemic sclerosis. Case report and review of the literature. Cleve Clin Q 1985;52:207-11.

23. Silman AJ. Pregnancy and scleroderma. Am J Reprod Immunol 1992;28:238-40.

24. Steen VD. Pregnancy in scleroderma. Rheum Dis Clin North Am 2007;33:345-58.

25. Chakravarty EF. Vascular complications of systemic sclerosis during pregnancy. Int J Rheumatol 2010;2010:287248.

26. Lidar M, Langevitz P. Pregnancy issues in scleroderma. Autoimmun Rev 2012;11:515-9.

27. Ibba-Maneschi L, Manetti M, Milia AF, Miniati I, Benelli G, Guiducci S, et al. Severe fibrotic changes and altered expression of angiogenic factors in maternal scleroderma. Ann Rheum Dis 2010;69:458-61.

28. Turk M, Pope JE. The frequency of scleroderma renal crisis over time: a metaanalysis. J Rheumatol 2016;43:1350-5.

29. Steen VD, Conte C, Day N, Ramsey-Goldman R, Medsger TA Jr. Pregnancy in women with systemic sclerosis. Arthritis Rheum 1989;32:151-7.

30. Gathiram P, Moodley J. Pre-eclampsia: its pathogenesis and pathophysiology. Cardiovasc J Afr 2016;27:71-8.

31. Subcommittee for scleroderma criteria of the American Rheumatism Association Diagnostic and Therapeutic Criteria Committee. Preliminary criteria for the classification of systemic sclerosis (scleroderma). Arthritis Rheum 1980;23:581-90.

32. van den Hoogen F, Khanna D, Fransen J, Johnson SR, Baron M, Tyndall A, et al. 2013 classification criteria for systemic sclerosis: an American College of Rheumatology/European League Against Rheumatism collaborative initiative. Ann Rheum Dis 2013; 72:1747-55. 